\title{
Borderline hypercholesterolaemia: when to introduce drugs
}

\author{
D. Bhatnagar and P.N. Durrington \\ University Department of Medicine, Manchester Royal Infirmary, Manchester, UK.
}

\section{Introduction}

'Please do not write any more articles about cholesterol and coronary disease and the diet and drugs which are supposed to influence them. The facts about coronary disease are these: the less atheromatous your ancestors, the harder your water, and the more habitual exercise you take, the less likely you are to be troubled by it. Do stop bothering about whether your fats are saturated or unsaturated, help yourselves liberally to butter and stop propagating these erroneous legends.'

This remains too frequently the view of medical practitioners in Britain. Sadly it must be one of the few statements of Richard Asher which does not remain as penetratingly accurate today as it was when first made: prescience had on this rare occasion deserted him. We still, of course, believe that susceptibility to coronary atheroma is in some individuals inherited, but this does not mean that their risk is immutable. Evidence that coronary disease morbidity and mortality can be decreased by therapy aimed at lowering serum cholesterol, is now strong. Indeed our patients are likely to benefit more from this than from many other medical practices, which are accepted without question. ${ }^{2-10}$ The debate concerning the "cholesterol hypothesis' has now moved on to consider how cholesterol or some factor closely related to its metabolism provokes atherogenesis and to determine the particular levels of cholesterol at which therapeutic intervention would be expected to produce benefit. ${ }^{11}$ It is this latter issue which is the subject of this article.

In some patients with hypercholesterolaemia (perhaps the minority) the decision to prescribe lipidlowering drug therapy, when diet has not produced a satisfactory decrease in the serum cholesterol, is easy. In others, however, it may involve a difficult clinical judgement. Indeed the majority of patients with hypercholesterolaemia must be regarded as borderline when drug therapy is to be considered. It is our purpose to provide a background of ideas, which will assist in making that decision. The origins and fate of the different lipoproteins which transport cholesterol are diverse, and it is naïve in the extreme to believe that any single numerical value for the serum cholesterol

Correspondence: P.N. Durrington, M.D., F.R.C.P.

Received: 14 February 1989 can stimulate a therapeutic reflex response without the proper diagnostic assessment of each individual patient. Supposing we limited ourselves to but a single therapeutic approach to hyponatraemia: what disasters would follow! Some knowledge of lipoprotein metabolism is essential to the clinician contemplating the mangement of hypercholesterolaemia.

\section{Lipoprotein physiology (Figure 1)}

The average Briton consumes almost $100 \mathrm{~g}$ of fat every day and much of this is triglyceride. The products of fat digestion are absorbed in the small intestine where they are synthesized into large triglyceride-rich lipoproteins called chylomicrons, which are secreted into the lacteals. They enter the blood circulation from the lymphatic system via the thoracic duct. As they circulate they come in contact with the enzyme, lipoprotein lipase, located in the capillary beds of tissues, such as muscle and adipose tissue, which have a high requirement for triglyceride as an energy source and as an energy store respectively. That enzyme releases fatty acids and glycerol from the chylomicron triglyceride and the lipoprotein particle becomes progressively smaller. Finally the small remnant particle, which is formed, is cleared by a special receptor on the liver cells, which recognizes one of the proteins present in the remnant called apolipoprotein E. This whole process is usually completed within a few hours of a meal and chylomicrons are not normally present in the plasma following an overnight fast.

Triglyceride is extremely important to any animal organism (except perhaps one living our present cossetted existence when our only movement is to be carried by car from one centrally heated, laboursaving building to another!). This is because triglyceride is a rich source of energy and is light and compact to store. Being a lipid it eschews water and thus the adipose cell comprises a triglyceride droplet with only a tiny rim of cytoplasm: no more than $15 \%$ of its weight is water and every gram of adipose tissue thus yields almost the full 9 Calories locked in each gram of triglyceride. Compare this with carbohydrate. Although refined carbohydrate contains 4 Calories per gram, because it is osmotically active, even substances

(C) The Fellowship of Postgraduate Medicine, 1989 


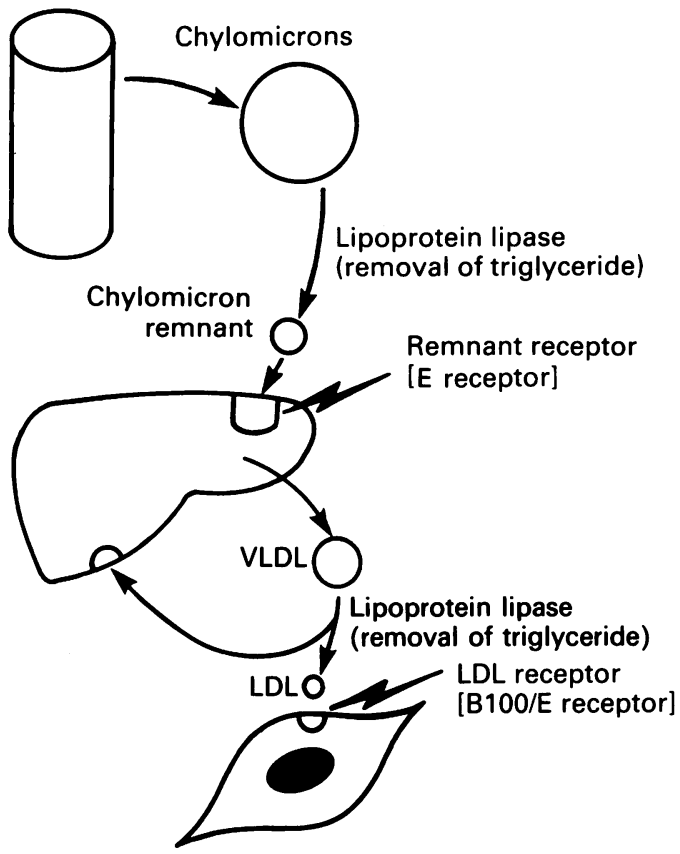

Figure 1 An outline of lipoprotein metabolism. Triglyceride-rich lipoproteins secreted by the gut (chylomicrons) and liver (VLDL) undergo lipolysis (lipoprotein lipase) as they circulate, resulting in the formation of chylomicron remnants and LDL, which together with some partially metabolized VLDL, are cleared by receptors on the liver and peripheral cells. Non-receptor mediated cell entry also occurs. HDL (not shown) probably participates in the return of excess cholesterol delivered to peripheral tissue back to the liver (reverse cholesterol transport).

such as glycogen can only be stored in limited amounts in any cell, meaning that the store of carbohydrate energy in a gram of muscle or liver is much less than one Calorie. The $70 \mathrm{~kg}$ man has about $15 \mathrm{~kg}$ of stored triglyceride representing 140,000 Calories (compare this to his $6 \mathrm{~kg}$ of protein equivalent to 24,000 Calories and $225 \mathrm{mg}$ of glycogen representing 900 Calories). Even with an energy expenditure of 2000 Calories per day these triglyceride stores would not be completely depleted after starvation for two months. This illustrates the very real difficulty experienced by patients, who are obese, in reducing their weight. To have an ideal weight of $70 \mathrm{~kg}$, but to be $20 \%$ overweight, is to have an extra $10 \mathrm{~kg}$ of triglyceride stored, which represents 90,000 Calories and many months of suffering, if it is to be removed!

The primal importance of triglyceride dictates that systems exist to transport it, at times other than after meals. The release from adipose tissue of nonesterified fatty acids, which can be directly respired by tissues, such as muscle, or converted to ketone bodies by the liver to act as respiratory substrates for other tissues, constitutes one transport system. The other is provided by the secretion of a triglyceride-rich lipoprotein by the liver, called very low density lipoprotein (VLDL). These lipoproteins, which are generally smaller than chylomicrons, are present in fasting plasma. Most of the triglyceride in a fasting blood sample is in VLDL. Within the circulation VLDL undergoes a similar sequence of events to the chylomicron: a progressive removal of its triglyceride load by the enzyme, lipoprotein lipase. This time the remnant particle, which is formed, is the low density lipoprotein (LDL). This lipoprotein is sufficiently small to cross the vascular endothelium and enter the extravascular extracellular fluid, where it comes in contact with all the cells of the body. It constitutes the system by which they receive cholesterol since it contains the cholesterol secreted by the liver as a component of VLDL, its precursor, and also cholesterol acquired during the circulation of VLDL and LDL from high density lipoprotein (HDL). Cholesterol is an essential component of cell membranes and every cell thus has a requirement for it. Certain specialized tissues also require cholesterol as a precursor for the synthesis of other sterols such as glucorticoids, mineralocorticoids, sex steroids, vitamin $D$ and bile salts. The cellular requirement for cholesterol is met by a membrane receptor, the LDL receptor, which recognizes LDL and allows its entry into the cell. Synthesis of the receptor is precisely regulated in response to the metabolic demands for cholesterol. In the liver, where the removal of cholesterol from the body is possible in the bile, this receptor-mediated removal of LDL from the circulation constitutes a major means of LDL catabolism. In addition to leaving the circulation by this means LDL may also exit by a non-receptor mediated route. This means of exit becomes increasingly significant as plasma LDL levels increase, because, unlike the receptor-mediated uptake of $L D L$, it is unregulated and remains concentration dependent. It can thus lead to the accumulation of excess cholesterol in the tissues and may contribute to atheroma.

Some four fifths of serum cholesterol is in LDL and about one fifth (more in women than men) is present in HDL. Cholesterol present in LDL is the reason for the positive relationship between total serum cholesterol and the risk of ischaemic heart disease (Figure 2). The HDL cholesterol concentration on the other hand is inversely related to risk. HDL is believed to have a key role in the removal of excess cholesterol from tissues and its return to the liver (reverse cholesterol transport). Unlike the triglyceride-rich lipoproteins it is secreted by the liver and gut as protein-rich particles, which contain little lipid. It is small and readily enters the tissue fluid, where it is the most abundant lipo- 


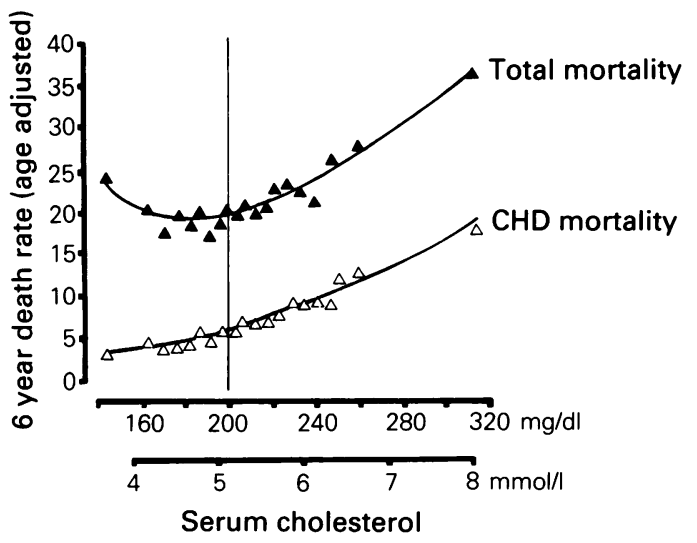

Figure 2 The relationship betwen cholesterol and mortality from coronary heart disease (CHD) and from all causes in 361, 662 men screened in the Multiple Risk Factor Intervention Study. ${ }^{20}$ The line at $200 \mathrm{mg} / \mathrm{dl}$ $(5.2 \mathrm{mmol} / \mathrm{l})$ indicates what is currently considered an ideal cholesterol level.

protein. After its secretion it acquires cholesterol, which it esterifies, and returns to the liver by passing it on to other lipoproteins or, perhaps in part, directly.

\section{Laboratory tests}

Most laboratories measure total serum cholesterol, total serum triglycerides and HDL cholesterol, and, if hypercholesterolaemia and/or hypertriglyceridaemia is present, will report which lipoproteins are increased according to the WHO classification (popularly known as the Fredrickson classification) (Table I). It is important not to regard this classification as a diagnostic classification since the different types may have a variety of causes both secondary and primary.

\section{Secondary cause of hyperlipoproteinaemia}

Those most frequently encountered are summarized in Table II. It is important to have fully excluded them, certainly by the stage when lipid lowering drug therapy is being considered. Sometimes adequate treatment of the primary disorder will result in resolution of the hyperlipidaemia, for example in hypothyroidism. Other disorders, such as diabetes, are important to recognize and treat, but their treatment will often not produce adequate control of the hyperlipidaemia (or reduce the risk of ischaemic heart disease) so that therapy specifically directed at the hyperlipidaemia is frequently still required. ${ }^{12}$ Drugs contributing to hyperlipidaemia, such as diuretics and $\beta$-adrenoreceptor blockers ${ }^{13,14}$ can present difficult decisions. We tend to avoid them in the management of hypertension in patients with hyperlipidaemia unless the blood pressure is inadequately controlled by other drugs. However, this approach would be considerably strengthened, if there was adequate evidence that some of the newer and better tolerated drugs, which can replace diuretics and $\beta$-blockers, actually do reduce mortality and morbidity from hypertension. In patients with established ischaemic heart disease we would only rarely allow the presence of hyperlipidaemia to influence other clinical considerations in the prescription of diuretic or $\beta$-blocking drugs.

\section{Primary hyperlipidaemia}

The British Hyperlipidaemia Association and the European Atherosclerosis Society have recommended that as a society we should attempt to reduce our mean cholesterol to around $5 \mathrm{mmol} / 1 .^{15,16}$ This recommendation is based on an appraisal of the epidemiological evidence linking cholesterol to ischaemic heart disease. In those nations where the mean cholesterol is below $5 \mathrm{mmol} / 1$ coronary heart disease is uncommon. ${ }^{17}$ In

Table I WHO classification of hyperlipoproteinaemia

\begin{tabular}{lllll}
\hline Type & $\begin{array}{l}\text { Lipoprotein } \\
\text { elevated }\end{array}$ & Cholesterol & Triglyceride & Frequency \\
\hline I & Chylomicrons* & Raised & Markedly raised & Very rare \\
IIa & LDL & Raised & Normal & Common \\
IIb & VLDL and LDL & Raised & Raised & Common \\
III & Beta VLDL & Raised & Raised & Rare \\
IV & VLDL & Normal & Raised & Common \\
V & Chylomicrons* & Raised & Markedly raised & Rare \\
& and VLDL & & & \\
\hline
\end{tabular}

\footnotetext{
* Milky appearance of plasma; LDL = low density lipoprotein; VLDL = very low density lipoprotein; beta VLDL = remnants of triglyceride-rich lipoprotein not normally present in any quantity.
} 
Table II Some common causes of secondary hyperlipidaemia
Obesity
Drugs e.g., $\beta$-blockers, thiazide diuretics
Diabetes mellitus
Alcohol
Renal disease
Hypothyroidism
Biliary obstruction
Myeloma

the UK the average cholesterol of middle-aged men is between 6 and $6.5 \mathrm{mmol} / \mathrm{l}^{18,19}$ with levels of about $0.5 \mathrm{mmol} / \mathrm{l}$ less in premenopausal women and $0.5 \mathrm{mmol} / 1$ greater afterwards. In a study of over 360,000 men in the USA it was established that there was no threshold below which ischaemic heart disease did not occur (Figure 2) ${ }^{20}$ However, total mortality reached a minimum at around $5 \mathrm{mmol} / \mathrm{l}$ and increased again as levels fell below $4 \mathrm{mmol} / \mathrm{l}$. This latter phenomenon was principally due to deaths from neoplasia within the first year or two of the cholesterol measurement and it is generally considered that the low cholesterol was caused by the malignancy present at the initial examinations. ${ }^{21}$ However, erring on the side of caution, ${ }^{22} 5 \mathrm{mmol} / \mathrm{l}$ rather than a lower level has been chosen as an ideal level.

This, of course, is not a realistic therapeutic goal for most patients with hyperlipidaemia and it is important therefore in planning their management to have some appreciation of the scale of their increased risk in order to balance it against any disadvantages and potential side effects of therapy. Both the British Hyperlipidaemia Association and the European Atherosclerosis Society after appraising the evidence from clinical trials, in particular the Lipid Research Clinics trial with cholestyramine, ${ }^{3,4}$ concluded that lipidlowering drugs should only exceptionally be considered unless the cholesterol level exceeded $6.5 \mathrm{mmol} / \mathrm{l}$ after a reasonable period of appropriate dietary modification. The results of the Helsinki Heart Study became available after these recommendations were published and it amply confirmed them by demonstrating decreased coronary morbidity and mortality without evidence of adverse side effects when men with cholesterol levels generally exceeding $6.4 \mathrm{mmol} / \mathrm{l}$, (actually non-HDL cholesterol $>5.2 \mathrm{mmol} / \mathrm{l}$, average HDL cholesterol $1.2 \mathrm{mmol} / \mathrm{l})$, despite diet, received gemfibrozil as opposed to placebo. ${ }^{7.8}$

It must, however, be emphasized that it has not been recommended that failure of the cholesterol level to fall below $6.5 \mathrm{mmol} / \mathrm{l}$ with dietary treatment is in itself an indication for lipid-lowering drug therapy: it is merely a starting point for its consideration. The risk of a serum cholesterol level greater than $6.5 \mathrm{mmol} / \mathrm{l}$ will vary tremendously from individual to individual.
Overall we can calculate from the Registrar General's statistics and a measure of the excess risk due to cholesterol $^{23}$ that some $2 \%$ of men whose cholesterol is less than $5 \mathrm{mmol} / \mathrm{l}$ will die by the age of 60 years and about $4 \%$ of those with cholesterol of $6 \mathrm{mmol} / 1$ and perhaps $6-8 \%$ of those at $7.5 \mathrm{mmol} / \mathrm{l}$. Thus, if the aim of treatment was to prevent death before the age of 60 , then even at a level of $7.5 \mathrm{mmol} / \mathrm{l}$ more than $90 \%$ of people receiving such treatment would derive no benefit from it and merely be exposed to its possible side effects. Of course, we realize that the prevention of coronary morbidity is also a major objective of cholesterol-lowering therapy and that the figures for combined mortality and morbidity before the age of 60 are about 3 times those for mortality alone. Also we realize that prevention of death from coronary disease before the age of 70 would be a more laudable objective. However, statistics for the likely benefits of cholesterol reduction after the age of 60 are more difficult to compute. The important point, however, is that the decision to use drugs in the management of hyperlipidaemia would be greatly facilitated, if we were able to predict with greater certainty whether the patient was one of those destined to succumb prematurely to coronary disease or one who would escape such a fate. Our clinical decision to introduce lipid-lowering drug therapy must therefore rely on an appraisal of the factors, which are likely to make each individual patient with hypercholesterolaemia more or less susceptible to accelerated atherogenesis. Factors critical to this are now considered (Table III).

Table III Factors which would encourage the prescription of lipid-lowering drugs in patients whose serum cholesterol exceeds $6.5 \mathrm{mmol} / \mathrm{l}$ despite dietary therapy

1. Genetic hyperlipidaemia

a) Familial hypercholesterolaemia

b) Type III hyperlipoproteinaemia

2. Adverse family history

3. Other coronary risk factors

a) Diabetes mellitus

b) Hypertension

c) Smoking history

4. Manifestation of coronary disease or peripheral arterial disease

5. Coronary artery bypass surgery

6. Hypercholesterolaemia combined with hypertriglyceridaemia and/or low HDL

7. Younger age

8. Male sex

\section{(i) The diagnosis}

Most hyperlipidaemia (types IIa, IIb and IV) occur as a result of environmental, particularly nutritional factors, interacting with some constitutional tendency 
to hyperlipidaemia, which is probably largely genetic. ${ }^{24}$ From inheritance studies and from the Gaussian distribution of the serum cholesterol concentration, this tendency is clearly polygenic in the majority of people with hyperlipidaemia. There are, however, some individuals whose hypercholesterolaemia does not originate in this way, but depends to a large extent on a single gene, and whose coronary risk may be very much higher. One clinical syndrome which exemplifies this is familial hypercholesterolaemia. ${ }^{25,26}$

\section{(a) Familial hypercholesterolaemia (FH)}

FH is a dominantly inherited condition. About 1 in 500 people in the UK are heterozygotes for FH. They generally have a type IIa or occasionally type IIb pattern and on average their serum cholesterol is more than $9 \mathrm{mmol} / \mathrm{l}$. However, there is considerable variation. In any patient with markedly elevated cholesterol levels it is important to consider $\mathrm{FH}$ as a possible diagnosis, but in the young, even when there is only borderline elevation of the cholesterol, FH should be considered as a cause. The reason for this is that there is a pronounced increase in coronary risk in $\mathrm{FH}$, which is not confined to those with the highest $\mathrm{LDL}$ cholesterol levels. ${ }^{27}$ Almost $60 \%$ of men and $15 \%$ of women with FH will die before the age of 60 years without treatment. ${ }^{28} \mathrm{FH}$ is due to a defect in the LDL receptor gene on chromosome 19. It is, of course, present throughout life and increased cholesterol can be detected in most affected children, unlike polygenic hypercholesterolaemia, which is often not detectable until the 3rd or even 4th decade. Most middle-aged patients with $\mathrm{FH}$ will have tendon xanthomata usually in the tendons of the dorsum of the hands and in the Achilles tendons. In younger adults the diagnosis may be difficult unless a first degree relative with tendon xanthomata is found or the cholesterol is known to have been raised in childhood. Death of a parent or sibling from ischaemic heart disease before the age of 50 is also strongly suggestive.

FH usually responds incompletely to diet and lipid-lowering drugs are almost invariably required and should not be withheld. The best therapeutic response is usually achieved with bile acid sequestrating agents (cholestyramine or colestipol) or the new HMG-CoA reductase inhibitors ${ }^{29}$ (Table IV). Fibrate drugs (gemfibrozil, bezafibrate, fenofibrate), probucol or nicotinic acid and its derivatives are sometimes helpful in addition.

\section{(b) Type III hyperlipoproteinaemia}

This is an unusual cause of hyperlipidaemia ${ }^{26,30}$ usually inherited as an autosomal recessive of variable penetrance. Both cholesterol and triglycerides are raised to
Table IV Lipid-lowering drugs

1. Bile acid sequestrants
e.g. cholestyramine, colestipol
2. Fibric acid derivatives
e.g. bezafibrate, fenofibrate, gemfibrozil
3. Probucol
4. Nicotinic acid and its derivatives
$\begin{gathered}\text { e.g. acipimox } \\ \text { 5. HMG-CoA reductase inhibitors } \\ \text { e.g. lovastatin, pravastatin, simvastatin }\end{gathered}$

about the same extent. The diagnosis may be confirmed in the laboratory by ultracentrifugation or identification of the patient's apolipoprotein $E$ phenotype. In many cases, however, the diagnosis may be made clinically by the presence of striate palmar xanthomata and/or tubero-eruptive xanthomata over the elbows and knees. The risk of coronary disease is similar to that in $\mathrm{FH}$, but there is also a marked increase in peripheral arterial disease. Type III may be responsive to diet. Any persisting (even borderline) elevation in cholesterol should, however, be treated with lipid-lowering drugs, of which the most effective are the fibrate drugs.

\section{(c) Familial combined hyperlipidaemia}

Studies of the families of patients, who have heart attacks in early life, have indicated the possible existence of a hyperlipidaemia with a variable phenotype producing type IIa, IIb or type IV hyperlipoproteinaemia in different members of the same family. ${ }^{31-36}$ It has been suggested that the condition, which has been called familial combined hyperlipidaemia $(\mathrm{FCH})$, is a dominant disorder. The evidence for this contention is inconclusive. The coronary risk in $\mathrm{FCH}$ is believed to be greater than for patients with polygenic hyperlipidaemia. $\mathrm{FCH}$ has been estimated to affect as many as 1 in 50 people, but since it has no specific clinical features it remains ill-defined. It does, however, emphasize the increased risk of hyperlipidaemia when it occurs in an individual with an adverse family history of coronary heart disease.

\section{(d) Particularly high cholesterol associated with the type IIa or IIb phenotype}

The relationship between cholesterol and the risk of ischaemic heart disease is exponential (Figure 2). It becomes progressively steeper. Thus levels of cholesterol exceeding $8.0 \mathrm{mmol} / \mathrm{l}$ after diet are associated with a sufficiently increased risk that, even if other adverse factors have not been found, they would generally justify cholesterol-lowering medication. $^{37}$ 


\section{(e) Other hyperlipidaemias}

In type IV hyperlipoproteinaemia, triglycerides in VLDL are raised, but LDL cholesterol levels are normal. There is no substantial evidence that treating type IV hyperlipoproteinaemia with drugs is beneficial. ${ }^{38}$ Laboratories which report patients with serum cholesterol levels in excess of $6.5 \mathrm{mmol} / \mathrm{l}$ as type IV should cease to do so and report them as type IIb hyperlipoproteinaemia to avoid confusion.

Occasionally patients have markedly raised chylomicron levels, even in the fasting state, usually in association with raised VLDL concentrations (type V hyperlipoproteinaemia) ${ }^{36,39}$ Their fasting triglyceride levels generally exceed $10 \mathrm{mmol} / \mathrm{l}$. Often the cholesterol level is above $6.5 \mathrm{mmol} / \mathrm{l}$, but this is not as a result of raised LDL levels, which are, in fact, low. The hypercholesterolaemia is due to cholesterol present in VLDL and chylomicrons. The condition may be asymptomatic, but there is a definite increased likelihood of acute pancreatitis, which is the usual justification for therapy. Unless there is coexistent diabetes an association with ischaemic heart disease is often not evident.

\section{(ii) Adverse family history}

An adverse family history is a risk factor for ischaemic heart disease. We usually regard this as the first manifestation of ischaemic heart disease in father or a brother before the age of 60 and in mother or sister before the age of 70 . Some familial aggregation of coronary disease must result from a shared environment and habits, such as smoking and diet. However, there is evidence for a true genetic influence on coronary disease susceptibility and, although this may operate to some extent through established risk factors such as hypercholesterolaemia, there is also an independent genetic effect, ${ }^{24,40}$ which will enhance susceptibility to other risk factors such as cholesterol. In practical terms this means that the decision to introduce lipid-lowering is more likely to be made when there is an adverse family history of coronary disease even when the diagnosis of FH has not been established.

\section{(iii) Other coronary risk factors}

It is clear from epidemiological investigations that whereas a single risk factor may have only a modest effect, in combination with others it may increase the likelihood of early-onset myocardial infarction manyfold $^{23,41}$ (Figure 3). Thus a hypertensive, diabetic, cigarette-smoker with hyperlipidaemia may be regarded as virtually certain of dying prematurely. It is

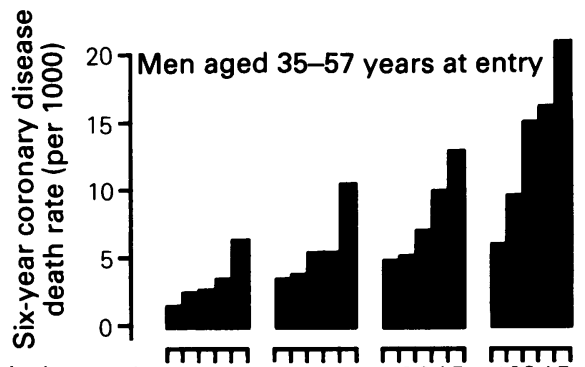

Serum cholesterol $\underbrace{12345} \underbrace{12345} \underbrace{12345} \underbrace{12345}$

(quintiles)

Diastolic blood $<90<90>90>90$ pressure $(\mathrm{mmHg})$

$$
\text { Smokers No No Yes Yes }
$$

Figure 3 The effect of progressively increasing concentrations of serum cholesterol on coronary risk alone and in combination with raised blood pressure in cigarettesmokers and non-smokers. Data from 356, 222 men screened in the Multiple Risk Factor Intervention Study. ${ }^{23}$

interesting that in Japan, where the averge cholesterol for middle-aged men is around $4 \mathrm{mmol} / \mathrm{l}$, coronary disease is uncommon even in smokers, and patients with diabetes and hypertension. ${ }^{17}$ The treatment of glycaemia or high blood pressure appears to have little impact on the increased incidence of ischaemic heart disease in diabetes and hypertension. This emphasizes the importance of considering cholesterol-lowering drug therapy in patients with these conditions, whose cholesterol persists above $6.5 \mathrm{mmol} / 1$ despite dietary treatment. ${ }^{14,42}$ Furthermore there can no longer be any justification for initiating treatment of diabetes or hypertension without also establishing whether hyperlipidaemia is present.

It is obvious that smokers should be advised to stop. However, even in those who do stop successfully, their past smoking history many continue to increase the likelihood of ischaemic heart disease for several years and may thus be another factor favouring drug therapy, if hypercholesterolaemia remains after dietary treatment.

\section{(iv) Manifestations of arteriosclerosis}

Patients with hyperlipidaemia, who have already developed clinical evidence of ischaemic heart disease or peripheral arterial disease, have declared themselves as being at particular risk from their hyperlipidaemia. Some authorities argue that treating their hyperlipidaemia at this stage is closing the stable door after its occupant has bolted, citing some depressing early trials of secondary prevention as 
justification for their opinion. ${ }^{43}$ This is, however, a view we are no longer able to share. Increasingly we are aware, as a result of coronary angiography, of patients who have sustained a myocardial infarction, but whose coronary disease has not spread throughout the coronary tree and who might yet benefit from treatment of their hyperlipidaemia. The outlook for patients following myocardial infarction has been transformed with the advent of cardioprotective drugs and of coronary artery bypass surgery. Except for the patient whose prognosis is poor because of heart failure, there seems little point in allowing their atheroma to proceed unchecked and we would be encouraged to introduce lipid-lowering drugs, where diet had been unsuccessful, in patients with hyperlipidaemia and manifestations of vascular disease. Recent evidence for this view comes from a successful secondary prevention study ${ }^{10}$ and from studies involving patients who had undergone coronary artery bypass surgery, in whom the rate of progress of atheroma in both the grafts and in the ungrafted coronary arteries was reduced by lipid-lowering therapy. ${ }^{9}$

\section{(v) Coronary artery bypass surgery}

Several studies have indicated that the main factor determining the rate at which disease progresses in grafts and ungrafted coronary arteries following bypass surgery is hyperlipoproteinaemia. ${ }^{44-46}$ For this reason we regard effective treatment of hyperlipidaemia, with drugs if necessary, as an essential part of post-operative management. Furthermore there can be little justification for making age any bar to such treatment or there would have been no point in attempting to preserve the patient by the operation. A placebo-controlled trial of two year's lipid-lowering treatment compared to placebo following coronary artery bypass surgery showed significantly less disease progression in the drug treated group assessed by coronary angiography. ${ }^{9}$ The initial average cholesterol level was $6.3 \mathrm{mmol} / 1$ and the level on treatment $4.7 \mathrm{mmol} / \mathrm{l}$. This study questions whether in selected groups of patients, such as those who have undergone coronary artery bypass surgery, the threshold for drug treatment of hypercholesterolaemia should be less than $6.5 \mathrm{mmol} / \mathrm{l}$. It is essential to remember, when assessing the presence of hypercholesterolaemia in patients following coronary artery bypass surgery, that the immediate effect of surgery, as is the case for myocardial infarction or acute coronary inefficiency, is to lower the cholesterol concentration. The serum cholesterol level should not be determined until at least 6 weeks have elapsed, if levels within the normal range are to be considered valid.

\section{(vi) Hypercholesterolaemia associated with hypertriglyceridaemia and/or low HDL}

The likelihood of a patient with hypercholesterolaemia developing ischaemic heart disease is increased if there is coexistent hypertriglyceridaemia (triglyceride $>2 \mathrm{mmol} / \mathrm{l}){ }^{47-50}$ Generally a decreased level of $\mathrm{HDL}$ cholesterol $(<0.9 \mathrm{mmol} / \mathrm{l})$ is also present when this occurs. There is no need for the clinician to be unduly concerned about the debate as to whether the increased risk results from the raised triglyceride or the low HDL: $:^{50.51}$ the essential point is that either is a marker of increased risk and there is thus a stronger indication for drug therapy when diet proves inadequate. Fibrate drugs which, in addition to decreasing cholesterol, lower triglycerides and raise HDL cholesterol, are the best first line therapy. Bile acid sequestrants may be added later to correct any remaining hypercholesterolaemia. If they are used alone, they tend to exacerbate the hypertriglyceridaemia and may not raise HDL.

Very occasionally patients are found whose total serum cholesterol is raised above $6.5 \mathrm{mmol} / \mathrm{l}$ due to high levels of HDL cholesterol. Under these circumstances drug treatment is inappropriate. The LDL cholesterol in such patients may be calculated from the following formula: LDL cholesterol $=$ total cholesterol - ([triglyceride $\div 2.2]+$ HDL cholesterol). Only patients with LDL cholesterol levels exceeding $4.5 \mathrm{mmol} / \mathrm{l}$ should be considered for further treatment. The formula is unsuitable for the calculation of LDL cholesterol when the serum triglyceride concentration exceeds $4.5 \mathrm{mmol} / 1$, but it is exceedingly unlikely that under those circumstances HDL would be increased; rather it would be expected to be low.

\section{(vii) Younger age}

When hypercholesterolaemia is discovered before the 4th or 5th decade it is a better determinant of risk than when detected in later life. ${ }^{23,41}$ Thus, for example, a 35 year old man whose cholesterol is $8.00 \mathrm{mmol} / \mathrm{l}$ will have about five times more likelihood of having a heart attack in the next 16 years than a man of the same age whose cholesterol is $5 \mathrm{mmol} / \mathrm{l}$. On the other hand, at the age of 60 the risk of the man with the higher cholesterol level would be only about 1.5 times that of the other man. It is important to interpret this information correctly. One reason for the change in relative risk is that the population of hypercholesterolaemic older men is much larger than than of young men. It has been swelled by men who have become hypercholesterolaemic with advancing age, ${ }^{26}$ whose risk is lower than those who have been hypercholesterolaemic since a young age. Therefore, although the clinician must look more critically at the 
decision to introduce lipid-lowering medication in older age groups, it must certainly not be dismissed, particularly when the history suggests that hypercholesterolaemia may have been present for a long while or there is an adverse family or personal history of vascular disease or other cardiovascular risk factors are present. Age is a major indicator of increased cardiovascular risk which is, of course, in itself immutable. However, to a large extent its influence may result from exposure to the cumulative action of other risk factors. It seems reasonable therefore to suppose that the earlier measures aimed at coronary prevention are adopted the greater will be the benefit, but that advice about diet and smoking, and in selected groups, the detection and treatment of hypertension and hyperlipidaemia may still have beneficial results, even in later life. In women similar considerations apply. There is often a fairly abrupt rise in cholesterol following the menopause. ${ }^{26}$ Although in general this is not associated with any abrupt change in their coronary rates, ${ }^{52}$ certain hyperlipidaemias requiring effective therapy, such as type III hyperlipoproteinaemia, are not, however, usually clinically evident until the menopause. ${ }^{30}$

\section{(viii) Male sex}

It has to be considered, in assessing the probable benefits of lipid-lowering therapy, that the likelihood of premature ischaemic heart disease in men is five to ten times that in women. In women with diabetes, however, this does not apply and their susceptibility approaches that of diabetic men. ${ }^{53}$ Women with FH or type III hyperlipoproteinaemia, although having a better prognosis than men with these conditions, are at greatly increased risk and lipid-lowering drug therapy should not be withheld on grounds of gender. The same also applies to many women with multiple risk factors. Furthermore in the authors' experience, a family history of premature ischaemic heart disease in a mother or sister is a particularly adverse factor both for men and women.

\section{Monitoring of response to drug and dietary treatment}

It is never justifiable to commence lipid-lowering medication on the basis of a single lipid determination. Dietary treatment should be the initial approach to cholesterol reduction and generally a minimum of two or three lipid measurements will be required over at least 3 or 4 months before the decision about lipidlowering medication should be taken. In patients, whose hyperlipidaemia is associated with obesity, longer may be required for satisfactory weight reduction and an adequate decrease in the cholesterol to have occurred. When medication is initiated the lipid levels should be monitored regularly until a satisfactory response is achieved. The effectiveness of fibrates can be assessed after one month. The dose of a bile acid sequestrant should be gradually built up starting with one sachet well soaked in fruit juice before breakfast, increasing to two sachets before breakfast after two weeks. After a further two weeks, lipid levels can be measured and, if necessary additional sachets added before dinner and occasionally lunch, at monthly intervals according to the cholesterol response. If nicotinic acid is used a similar gradual build up to an effective dose is required. Probucol takes at least 3 months to become fully effective.

When a satisfactory response has been achieved the lipid levels should be checked every six months or annually. It is usually important to continue to check the lipids every year or so even in patients, who continue with diet alone and occasionally to revise the decision to withhold lipid-lowering medication. As far as possible, the treatment of patients with hyperlipidaemia should be undertaken by the general practitioner with the hospital-based lipid clinic providing treatment and further investigation of the more complicated or high risk disorders and providing a second opinion about whether to introduce lipid-lowering medication or to continue with diet alone in some borderline cases.

\section{Conclusions}

Lipid-lowering medication may be considered in patients whose serum cholesterol exceeds $6.5 \mathrm{mmol} / \mathrm{l}$ despite diet (occasionally at lower levels in patients who have undergone coronary artery surgery). As precise a diagnosis as possible should be made before making the decision. All patients should have fasting serum cholesterol, triglyceride and HDL cholesterol measured. Urinalysis, serum creatinine (or urea), liver enzymes, blood glucose and sometimes serum thyroxine and occasionally immunoglobulins are required to detect secondary causes of hyperlipidaemia. Patients considered to have familial hypercholesterolaemia or type III hyperlipoproteinaemia, whose cholesterol level is persistently elevated, should generally receive lipid-lowering drugs. The decision to prescribe these agents in other patients is based on an assessment of their individual risk. This takes into account factors such as family history of premature arteriosclerosis, personal history of arteriosclerosis, coronary artery surgery, diabetes mellitus, smoking habit and hypertension. Associated hypertriglyceridaemia or low serum HDL cholesterol levels are also important considerations and so is the height of the cholesterol level. Treatment is more strongly indicated in younger age groups and in men. 
Bile acid sequestrating agents should be the drug of first choice when marked hypercholesterolaemia is present except in patients with associated hypertriglyceridaemia when fibrate drugs should be the first line therapy. Fibrates may also be useful for less marked hypercholesterolaemia, particularly if there is coexistent hypertriglyceridaemia, and as an adjunct to bile acid sequestrant therapy. Fibrates are also the usual first line therapy for diabetic hyperlipidaemia, unless significant nephropathy is present. Nicotinic acid and its derivatives and probucol are occasionally helpful. The advent of HMG-CoA reductase inhibitor drugs is expected to provide a further group of agents, which will effectively lower cholesterol and are also proving

\section{References}

1. Asher, R. Six honest serving men for medical writers. In: Avery Jones, F. (ed.) Richard Asher Talking Sense. Pitman Medical, London, 1972, pp. 54-69.

2. Thompson, G.R. Evidence that lowering serum lipids favourably influences coronary heart disease. $Q \mathrm{~J} \mathrm{Med}$ 1987, 238: 87-95.

3. Lipid Research Clinics Program. The Lipid Research Clinics Coronary Primary Prevention Trial Results 1. Reduction in incidence of coronary heart disease. JAMA 1984, 251: $351-364$.

4. Lipid Research Clinics Program. The Lipid Research Clinics Coronary Primary Prevention Trial Results II. The relationship of reduction in incidence of coronary heart disease to cholesterol lowering. JAMA 1984, 251: 365-374.

5. Grundy, S.M. Cholesterol and coronary heart disease. A new era. JAMA 1986, 256: 2849-2858.

6. Lewis, B., Mann, J.I. \& Mancini, M. Reducing the risks of coronary heart disease in individuals and in the population. Lancet 1986, i: 956-959.

7. Frick, M.H., Elo, O., Haapa, K. et al. Helsinki Heart Study, Primary-prevention trial with gemfibrozil in middle-aged men with dyslipidemia. Safety of treatment, changes in risk factors and incidence of coronary heart disease. N Engl J Med 1987, 317: 1237-1245.

8. Manninen, V., Elo, O., Frick, M.H. et al. Lipid alterations and decline in the incidence of coronary heart disease in the Helsinki Heart Study. JAMA 1988, 260: $641-561$

9. Blankenhorn, D.H., Nessim, S.A., Johnson, R.L. et al. Beneficial effects of combined colestipol-niacin therapy on coronary atherosclerosis and coronary venous bypass grafts. JAMA 1987, 257: 3233-3240.

10. Carlson, L.A. \& Rosenhamer, G. Reduction of mortality in the Stockholm Ischaemic Heart Disease Secondary Prevention Study by combined treatment with clofibrate and nicotinic acid. Acta Med Scand 1988, 223, 405-418.

11. Steinberg, D. Lipoproteins and atherosclerosis. A look back and a look ahead. Arteriosclerosis 1983, 3: 283-301.

12. Winocour, P.H., Durrington, P.N., Ishola, M. et al. The prevalence of hyperlipidaemia and related clinical features in insulin-dependent diabetes mellitus. $Q J \mathrm{Med}$ 1989, 70: (in press). to have a moderate triglyceride-lowering action.

'There is no mystery about why the incidence of vascular disease, like that of bronchial cancer and of venereal disease, continues to rise for many decades after pathogenesis is established. Human beings, including physicians and informed laymen, are eager for excuses not to face annoying facts . . . ${ }^{54}$ Let us now grasp the nettle.

\section{Acknowledgement}

We are grateful to Miss J. Rogerson for expert secretarial assistance.

13. Durrington, P.N., Brownlee, W.C. \& Large, D.M. Effects of $\beta$-adrenoceptor blocking drugs with and without cardioselectivity and intrinsic sympathomimetic activity on lipoprotein metabolism in hypertriglyceridaemic patients and normal men. Clin Sci 1985, 69: $713-719$.

14. Grimm, R.H. \& Hunninghake, D.B. 1986 Lipids and hypertension. Implications of new guidelines for cholesterol management in the treatment of hypertension. Am J Med 1986, 80(2A): 56-63.

15. Shepherd, J., Betteridge, D.J., Durrington, P.N. et al. Strategies for reducing coronary heart disease and desirable limits for blood lipid concentrations: guidelines of the British Hyperlipidaemia Association. Br Med J 1987, 295: $1245-1246$.

16. Study Group of the European Atherosclerosis Society. Strategies for the prevention of coronary heart disease: a policy statement of the European Atherosclerosis Society. Eur Heart J 1987, 8: 77-88.

17. Keys, A. Coronary heart disease - the global picture. Atherosclerosis 1975, 22: 149-192.

18. Thelle, D.S., Shaper, A.G., Whitehead, T.P. et al. Blood lipids in middle-aged British men. Br Heart J 1983, 49: 205-213.

19. Mann, J.I., Lewis, B., Shepherd, J. et al. Blood lipid concentrations and other cardiovascular risk factors: distribution, prevalence, and detection in Britain. $\mathrm{Br}$ Med J 1988, 296: 1702-1706.

20. Martin, M.H., Hulley, S.B., Browner, W.S. et al. Serum cholesterol, blood pressure and mortality: implications from a cohort of 361, 662 men. Lancet 1986, ii: 933-936.

21. Sherwin, R.W., Wentworth, D.N., Dutler, J.A. et al. Serum cholesterol levels and cancer mortality in 361, 662 men screened for Multiple Risk Factor Intervention Trial. JAMA 1987, 257: 943-948.

22. Schatzkin, A., Hoover, R.N., Taylor, P.R. et al. Serum cholesterol and cancer in the NHANES 1 epidemiologic follow up study. Lancet 1987, ii: 298-301.

23. Stamler, J., Wentworth, D. \& Neaton, J.D. Is relationship between serum cholesterol and risk of premature death from coronary heart disease continuous and graded? Finding in 356, 222 primary screens of the Multiple Risk Factor Intevention Trial (MRFIT). JAMA 1986, 256: 2823-2328. 
24. Burn, J. Durrington, P.N. \& Harris, R. Genetics and cardiovascular disease. In Rowlands, D.J. (ed.) Recent Advances in Cardiology, Vol. 10. Churchill Livingstone, Edinburgh, 1987, pp. 27-47.

25. Goldstein, J.L. \& Brown, M.S. Familial hypercholesterolaemia. In: Stanbury, J.B., Wyngaarden, J.B., Fredrickson, D.S., Goldstein, J.L. \& Brown, M.S. (eds) The Metabolic Basis of Inherited Disease. McGraw-Hill, New York, 1983, pp. 672-712.

26. Durrington, P.N. \& Hyperlipidaemia, Diagnosis and Management. Butterworths Scientific, London, 1989.

27. Miettinen, T.H. \& Gytling, H. Mortality and cholesterol metabolism in familial hypercholesterolaemia. Long term follow up of 96 patients. Arteriosclerosis 1988, 8: $163-167$.

28. Slack, J. Risks of ischaemic heart disease in familial hyperlipoproteinaemia states. Lancet 1969, ii: $1380-1382$.

29. Havel, R.J., Hunninghake, D.B., Illingworth, D.R. et al. Lovastatin (mevinolin) in the treatment of heterozygous familial hypercholesterolaemia. A health centre study. Ann Intern Med 1987, 107: 609-615.

30. Goldstein, J. \& Brown, M.S. Familial type 3 hyperlipoproteinaemia (dysbetalipoproteinaemia). In: Stanbury, J.B., Wyngaarden, J.B., Fredrickson, D.S., Goldstein, J.L. \& Brown, M.S. (eds) The Metabolic Basis of Inherited Disease. McGraw-Hill, New York, 1983, pp. 655-671.

31. Goldstein, J.L., Hazzard, W.R., Schrott, H.G. et al. Hyperlipidemia in coronary heart disease I Lipid levels in 500 survivors of myocardial infarction. J Clin Invest 1973, 52: 1533-1543.

32. Goldstein, J.L., Hazzard, W.R., Schrott, H.G. et al. Hyperlipidemia in coronary heart disease II Genetic analysis of lipid levels in 176 families and delineation of a new inherited disorder, combined hyperlipidemia. J Clin Invest 1973, 52: 1544-1568.

33. Nikkila, E.A. \& Aro, A. Family study of serum lipids and lipoproteins in coronary heart disease. Lancet 1973, i: 954-958.

34. Slack, J. The genetic contribution to coronary heart disease through lipoprotein concentrations. Postgrad Med J 1975, 51 (suppl 8): 27-32.

35. Hazzard, W.R., Goldstein, J.L., Schrott, H.G. et al. Hyperlipidaemia of coronary heart disease III Evaluation of lipoprotein phenotypes of 156 genetically defined survivors of myocardial infarction. J Clin Invest 1973, 52: 1569-1577.

36. Grundy, S.M., Chait, A. \& Brunzell, J.D. 1987 Familial combined hyperlipidaemia workshop. Arteriosclerosis 1987, 7: 203-207.

37. British Cardiac Society Working Group on Coronary Prevention. Conclusions and recommendations. $\mathrm{Br}$ Heart $J$ 1987, 57: 188-189.

38. Consensus Conference. Treatment of hypertrigly-

39. Nikkila, E.A. Familial type 5 hyperlipoproteinaemia. In: Stanbury, J.B., Wyngaarden, J.B., Fredrickson, D.S., Goldstein, J.L. \& Brown, M.S. (eds) The Metabolic Basis of Interited Disease. McGraw-Hill, New York, 1983, pp. 634-638.
40. Jorde, L.B. \& Williams, R.R. Relation between family history of coronary artery disease and coronary risk variables. Am J Cardiol 1988, 62: 708-713.

41. Dawber, T.R. The Framingham Study. The Epidemiology of Atherosclerotic Disease. Harvard University Press, Cambridge, Massachusetts, 1980.

42. Durrington, P.N. \& Winocour, P.H. Therapeutic aspects of hyperlipidaemia in diabetes. Postgrad Med J 1989, 65 (Suppl 1): (in press).

43. Oliver, M.F. Serum cholesterol. The knave of hearts and the joker. Lancet 1981, ii: 1090-1095.

44. Palac, R.T., Meadows, W.R., Hwang, M.H. et al. Risk factors related to progressive narrowing in aortocoronary vein grafts studied 1 and 5 years after surgery. Circulation 1982, 66 (suppl 1): 140-144.

45. Campeau, L., Enjalbert, M., Lesperance, J. et al. The relation of risk factors to the development of atherosclerosis in saphenous-vein bypass grafts and the progression of disease in the native circulation. A study 10 years after aortocoronary bypass surgery. $N$ Engl J Med 1984, 311: 1329-1332.

46. Fox, M.H., Gruchow, H.W., Barboriak, J.J. et al. Risk factors among patients undergoing repeat aortocoronary bypass procedures. J Thorac Cardiovasc Surg 1987, 93: 56-61.

47. Carlson, L.A. \& Bottiger, L.E. Ischaemic heart disease in relation to fasting values of plasma triglycerides and cholesterol. Stockholm Prospective Study. Lancet 1972, i: $865-868$.

48. Cabin, H. \& Roberts, W.C. Relation of serum total cholesterol and triglyceride levels to the amount and extent of coronary arterial narrowing by atherosclerotic plaque in CHD. Am J Med 1982, 73: 227-234.

49. Moorjani, S., Gagne, C., Lupien, P.J. \& Brunn, D. Plasma triglyceride related decrease in high-density lipoprotein cholesterol and its association with myocardial infarction in heterozygous familial hypercholesterolaemia. Metabolism 1986, 35: 311-316.

50. Castelli, W.P. The triglyceride issue: a view from Framingham. Am Heart $J$ 1986, 112: 432-437.

51. Hulley, S.B., Rosenman, R.H., Bahol, R.D. \& Brand, R.I. Epidemiology as a guide to clinical decision. The association between triglyceride and coronary heart disease. $N$ Engl J Med 1980, 302: 1383-1389.

52. Godsland, I.F., Wynn, V. Crook, D. \& Miller, N.E. Sex, plasma lipoprotein and atherosclerosis. Prevailing assumptions and outstanding questions. Am Heart J 1987, 114: 1467-1503.

53. Jarrett, J. Diabetes and the heart: coronary heart disease. Clin Endocrinol Metab 1978, 6: 389-402.

54. Dock, W. Atherosclerosis. Why do we pretend the pathogenesis is mysterious? Circulation 1974, 50: 647-649. 